\title{
Frequency-specific coupling between trial-to-trial fluctuations of neural responses and response-time variability
}

\author{
Nicoletta Adamo · Sarah Baumeister • Sarah Hohmann • \\ Isabella Wolf • Nathalie Holz • Regina Boecker • Manfred Laucht • \\ Tobias Banaschewski • Daniel Brandeis
}

Received: 12 August 2014/Accepted: 13 February 2015/Published online: 28 February 2015

(C) Springer-Verlag Wien 2015

\begin{abstract}
We assessed intra-individual variability of response times (RT) and single-trial P3 amplitudes following targets in healthy adults during a Flanker/NO-GO task. RT variability and variability of the neural responses coupled at the faster frequencies examined $(0.07-0.17 \mathrm{~Hz})$ at $\mathrm{Pz}$, the target-P3 maxima, despite non-significant associations for overall variability (standard deviation, SD). Frequencyspecific patterns of variability in the single-trial P3 may help to understand the neurophysiology of RT variability and its explanatory models of attention allocation deficits beyond intra-individual variability summary indices such as $\mathrm{SD}$.
\end{abstract}

Keywords Intra-individual response-time variability . Event-related potential $\cdot$ Cognitive control .

Attention deficit

N. Adamo $\cdot$ S. Baumeister $\cdot$ S. Hohmann $\cdot$ I. Wolf $\cdot$ N. Holz . R. Boecker · M. Laucht · T. Banaschewski - D. Brandeis ( $\square)$ Department of Child and Adolescent Psychiatry and

Psychotherapy, Central Institute of Mental Health, Medical Faculty Mannheim/Heidelberg University, Mannheim, Germany

e-mail: daniel.brandeis@zi-mannheim.de

\section{N. Adamo}

National Health Service (NHS) Tayside, Child and Adolescent Mental Health Service, Dundee, UK

I. Wolf

Department of Neuroimaging, Central Institute of Mental Health, Medical Faculty Mannheim/Heidelberg University, Mannheim, Germany

M. Laucht

Department of Psychology, Division of Clinical Psychology,

University of Potsdam, Potsdam, Germany

\section{Introduction}

Elevated intra-individual response-time variability (RTV), reflecting inconsistent behavioral responses in cognitive performance, has been reported in individuals with psychiatric conditions, most commonly, attention-deficit/hyperactivity disorder (ADHD) (Kofler et al. 2013), as well as in healthy aging, neurological and neurodegenerative disorders (MacDonald et al. 2006). Research investigating RTV has yet to clarify the underlying construct and neural underpinnings of this cognitive measure.

Studies examining the RT distribution have shown that high RTV is largely determined by a few slow responses and may reflect periodic lapses in attention (Leth-Steensen et al. 2000; West et al. 2002). Consistent with models of impaired regulation of 'top-down' attentional

\author{
D. Brandeis \\ Department of Child and Adolescent Psychiatry, \\ University of Zurich, Zurich, Switzerland \\ D. Brandeis \\ Center for Integrative Human Physiology, University of Zurich, \\ Zurich, Switzerland \\ D. Brandeis \\ Neuroscience Center Zurich, University of Zurich and ETH \\ Zurich, Zurich, Switzerland
}


control underlying RTV (Stuss et al. 2003), these data supported the hypothesis that RTV reflects deficient attention allocation to meet task demands. Accordingly, studies have used the excellent temporal resolution of electroencephalography (EEG) to test the association between RTV and event-related potentials, particularly the parietal P3 following target stimuli (target-P3) thought to reflect attention allocation. Specifically, reduced target-P3 mean amplitude has been observed in healthy adults (Saville et al. 2011) and individuals with ADHD (Banaschewski et al. 2004; Kratz et al. 2011) exhibiting high RTV. An inverse relationship between RTV and target-P3 amplitude has also been reported in healthy adults (Nakata et al. 2012) and individuals with traumatic brain injury (Segalowitz et al. 1997).

Electrophysiological studies have also begun using single-trial information to examine the underlying neurophysiology of RTV. One early study related higher target-P3 amplitude variability, measured as the standard deviation of the mean (SD), to greater SD of RT (SDRT), commonly used to measure RTV (Lazzaro et al. 1997). In contrast, recent work indicated less variable target-P3 amplitudes in individuals with high RTV and no correlation between SD of target-P3 and SD-RT (Saville et al. 2011). Therefore, the relationship between RTV and variability of the target-P3 amplitude remains unclear.

However, SD-RT alone does not measure characteristic periodic dynamics of RTV (Castellanos et al. 2005). Frequency analyses indicate that RTV occurs at slow cycle lengths (Castellanos et al. 2005; Feige et al. 2013), suggesting that frequency-specific RT fluctuations may help link RTV to its underlying neurophysiological mechanisms (Sonuga-Barke and Castellanos 2007). Accordingly, we tested whether overall and frequency-specific fluctuations of target-P3 amplitudes relate to RT fluctuations.

\section{Methods}

Participants

We collected RT data during simultaneous EEG recording and functional magnetic resonance imaging (fMRI) in a Flanker/NO-GO task from 23 healthy participants (12 males) aged $20-35$ years $(M=24.70 \pm 4.29)$. Here, analyses on RT and EEG variability are presented; EEG-fMRI results have been reported previously (Baumeister et al. 2014). Due to insufficient EEG data quality, six participants were excluded from subsequent analyses, leaving a final sample of 17 participants $(9$ males; $M=24.7$ \pm 4.1 years) who attained above $90 \%$ accuracy. All subjects gave written informed consent prior to their participation. The study was approved by the Ethics Committee of the Ruprecht-Karls-University Heidelberg Medical Faculty.

\section{Experimental paradigm}

All participants performed a 10-min, 19-s long Flanker/NOGO task (Baumeister et al. 2014; Blasi et al. 2006). Briefly, 145 stimuli, with three "GO" (neutral, congruent, incongruent) and one "NO-GO" conditions, were pseudorandomly presented for $800 \mathrm{~ms}$ interleaved with a fixation cross. Participants were instructed to press a button as fast and accurately as possible according to the direction of the target arrow while ignoring the two flankers on each side during "GO" conditions (e.g., $>><>>$ or $\square \square>\square \square$ ), but inhibit their response for "NO-GOs" $(\mathrm{XX}>\mathrm{XX})$. For 109 trials the inter-stimulus interval (ISI) was 3-s. To introduce variable 3-12-s ISI, the remaining trials were followed by up to three 3-s null events presented in pseudorandom order, during which the fixation cross continued.

\section{Electrophysiological recording and analysis}

The signals were recorded from 60 EEG electrodes (Plichta et al. 2013), 2 electrooculogram and 2 electrocardiogram electrodes using MR-compatible caps and amplifiers (Brain Products, Gilching, Germany) and a $0.01-250 \mathrm{~Hz}$ recording bandpass. Standard fMRI gradient, ballistocardiogram and eye movement correction, plus $30 \mathrm{~Hz}$ low-pass filtering and re-referencing to the average reference are described in Baumeister et al. (2014). The target-P3 analysis segment was $1500 \mathrm{~ms}$ including a 250-ms pre-stimulus period. Baseline-corrected, artifact-free epochs were averaged for each target condition. For the single-trial analysis, we exported the mean voltage of the 300-600-ms poststimulus window for each epoch at $\mathrm{Pz}$ and $\mathrm{Cz}$ (similar to the single-trial P3 analyses in Baumeister et al. 2014 but focusing on the GO-P3, and consistent with Lazzaro et al. 1997; Nakata et al. 2012).

Variability measures

We computed the intra-individual SD-RT and SD from single-trial target-P3 amplitudes (target-P3 SD) for correct responses after excluding the task's first $30 \mathrm{~s}$ to avoid potential adjustment effects. To obtain continuous RT and target-P3 time series, we replaced missing responses, NOGO and null events using linear interpolation between adjacent observations. Expected trial-type effects on mean RT (data not shown) and mean target-P3 amplitude (Fig. 1a) were removed by linear regression yielding residual time series. We applied the Morlet-wavelet transform to each participant's RT and target-P3 time series as 


\section{A}

Target-P3 maps

$(300-600 \mathrm{~ms})$

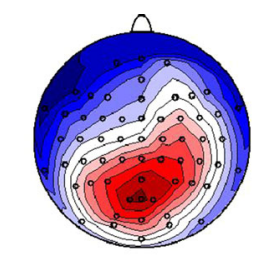

Incongruent

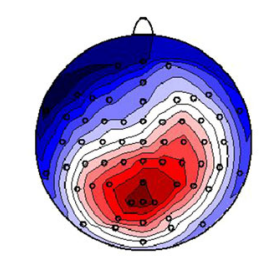

Congruent

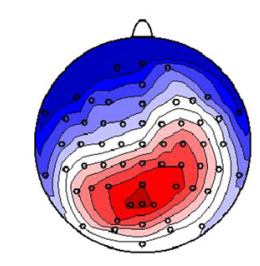

Neutral

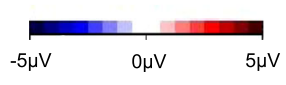

$\mathrm{Pz}$

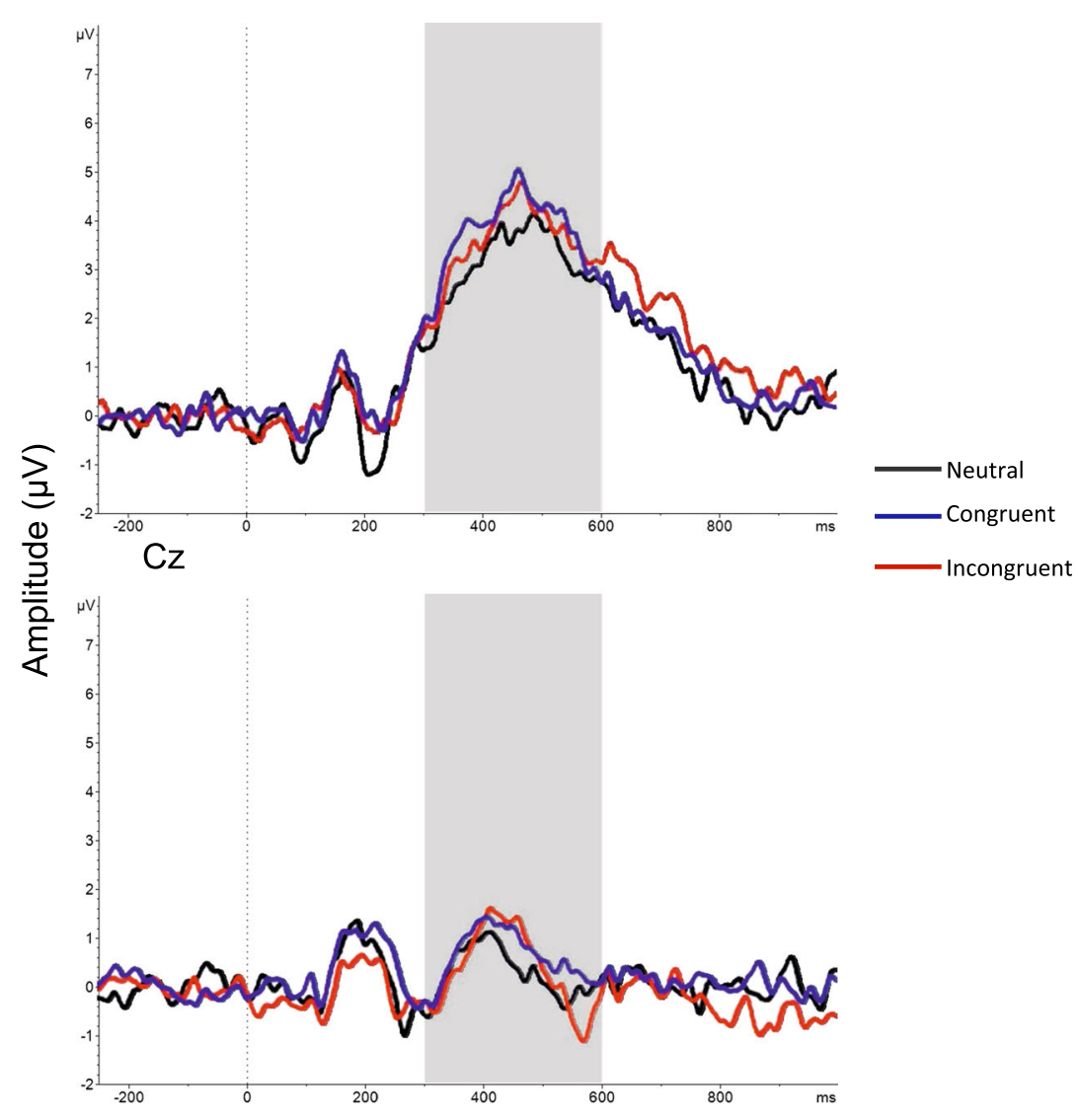

B

Response times

Slow-5

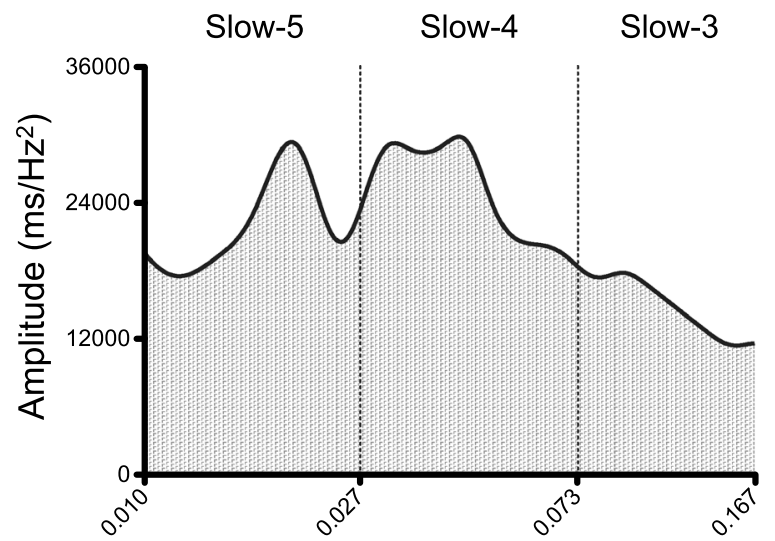

Target-P3

Slow-5

Slow-4

Slow-3

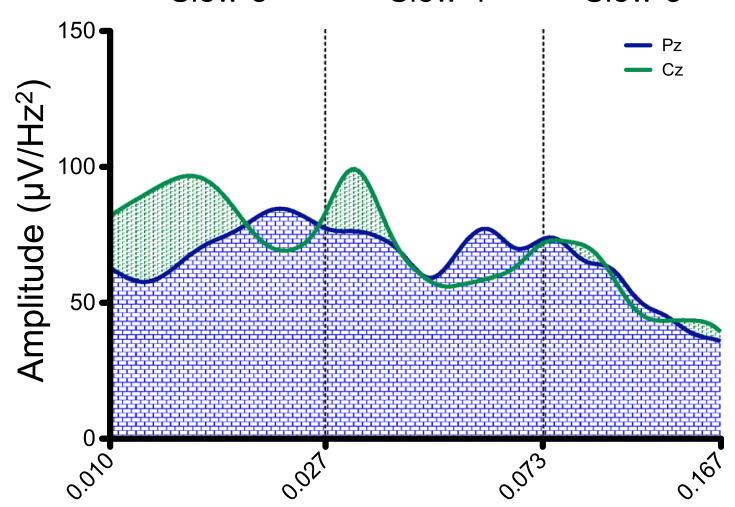

Frequency $(\mathrm{Hz})$
Fig. 1 a Maps of the grand mean in the selected post-stimulus window and stimulus-locked waveforms of the target-P3 for the "GO" conditions; congruent $>$ neutral, $t=3.6, p<0.01$. b Average

described previously for RT data (Adamo et al. 2012; Di Martino et al. 2008). Lastly, we estimated the average amplitude of three frequency bands based on physiological amplitude of the sampled frequency spectrum $(0.006-0.167 \mathrm{~Hz})$ across participants for RT fluctuations (left panel) and target-P3 fluctuations at $\mathrm{Pz}$ and $\mathrm{Cz}$ (right panel)

models of slow brain oscillations (Penttonen and Buzsaki 2003): Slow-5 (0.010-0.027 Hz), Slow-4 (0.027-0.073 Hz) and Slow-3 (0.073-0.167 Hz). 
Fig. 2 Correlations between RTV and target-P3 amplitude variability. $R T$ response time, $S D$ standard deviation. Upper and lower boxes display the correlations between SD-RT $(y$ axis) and target-P3 SD ( $x$-axis), and between wavelet transformbased amplitudes for RT fluctuations ( $y$-axis) and targetP3 fluctuations ( $x$-axis), respectively
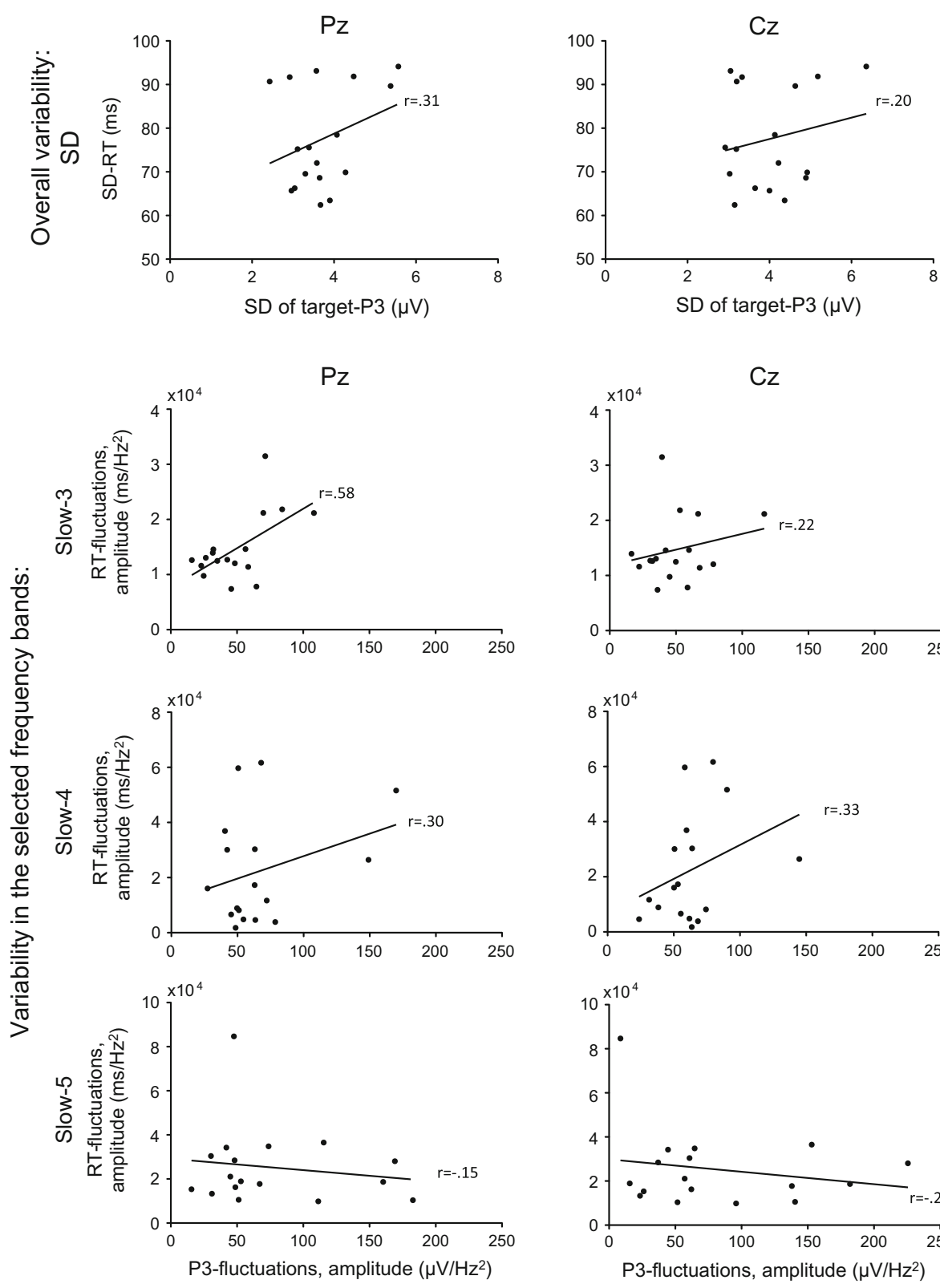
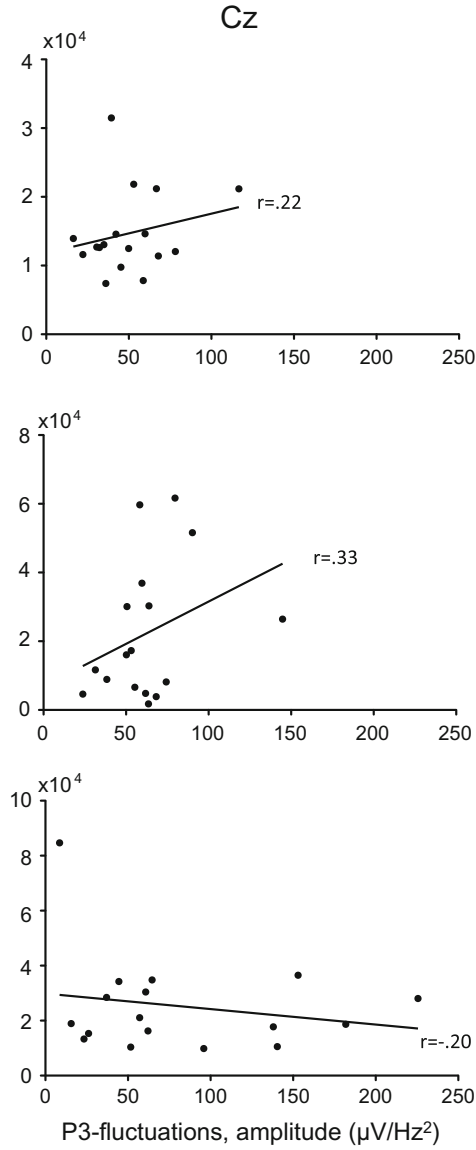

Statistical analyses

We conducted Pearson's correlations to measure the relationship between all variability measures (SD and frequency amplitudes) of RT performance and the corresponding indices for the target-P3 amplitude at both $\mathrm{Pz}$ and $\mathrm{Cz}$.

\section{Results}

The relationships between RTV and target-P3 variability indices are depicted in Fig. 2. SD-RT did not correlate with the target-P3 SD at Pz and Cz. The RT fluctuations positively correlated with target-P3 fluctuations in the frequencies
$0.073-0.17 \mathrm{~Hz}$ (Slow-3) at $\mathrm{Pz} \quad(r=0.58, \quad p=0.014$, $p<0.05$ after Bonferroni correction for three bands), but not at $\mathrm{Cz}$. At both sites, we did not find significant correlations between behavioral and neural responses for frequencies in the Slow-4 and Slow-5 bands. Still, these slower frequency bands contributed at least as much to the absolute variability in RT and target-P3 as the Slow-3 band (Fig. 1b), indicating that the correlation in the Slow-3 band did not simply follow the main sources of variability.

\section{Discussion}

Our aim was to determine whether trial-to-trial variability of target-P3 amplitude reflects RTV and if their 
correlations occur at specific frequencies. While RTV and target-P3 variability did not correlate when measured with the SD, we observed a frequency-specific coupling between behavior and neural responses in the Slow-3 frequency band $(0.073-0.17 \mathrm{~Hz})$ at the electrode Pz.

Our findings parallel those of a study showing a decoupling between SD-RT and SD of target-P3 amplitude (Saville et al. 2011), but contrast a recent report of a positive association between SD-RT and target-P3 SD at Pz (Moore et al. 2013). Specifically, Moore and colleagues found that SD-RT and target-P3 SD correlated in a flanker-incompatible condition (i.e., participants must respond to the opposite direction of the target stimulus) but not in the compatible condition in children. Here, we neither found significant correlations between modalities for the SD across congruent, incongruent and neutral stimuli (details in "Results") nor for each condition separately (data not shown). Beyond possible reduced power in our small sample, the uncoupling between SD indices of behavioral and target-P3 responses might result from lower cognitive control demand in our paradigm with flanker-compatible conditions only and better cognitive control in our age group.

Increasing evidence has indicated that RTV occurs at slow frequencies (e.g., $<0.25 \mathrm{~Hz}$ ). As RT fluctuation patterns resemble the spontaneous hemodynamic fluctuations at brain areas implicated in attention impairments (Fox and Raichle 2007; Zuo et al. 2010), hypotheses suggest that frequency-specific measures may help understand the underlying pathophysiology of RTV and the associated attention deficits (Sonuga-Barke and Castellanos 2007). Accordingly, we extended the study of the association between RTV and target-P3 variability to their underlying trial-to-trial fluctuations. Supporting the hypothesis that greater target-P3 variability reflects higher RTV (Lazzaro et al. 1997; Moore et al. 2013), our findings indicate that slow periodic variability patterns may identify such an association regardless of the correlation with SD.

Our study has a number of limitations. To conduct frequency analyses, interpolation of nearly half of the time series and interspersed gaps of $\geq 3$-s might potentially enhance frequencies in the Slow-3 range. However, data manipulation would have affected the results for Pz- and Cz-derived fluctuations equally. Replications in larger sample sizes are needed to confirm these preliminary findings. Yet, prior evidence suggesting that neural fluctuations in relatively fast frequencies can be estimated with better accuracy supports our findings of neurophysiological oscillations at similar frequencies (Fornito et al. 2011). Finally, future analyses are warranted to assess further potential accounts of RTV, including variability of target-P3 latency (Saville et al. 2011) and prestimulus state as measured with EEG spectral power (Reinhart et al. 2011).
In conclusion, we found that RTV relates to target-P3 variability in specific frequencies, and confirmed the association between RTV and slow fluctuations in attention allocation measured with the target-P3, despite non-significant associations for overall variability (SD). We suggest that frequency-specific trial-to-trial fluctuations are important to detect and understand the relationship between neural and behavioral variability.

\section{References}

Adamo N, Di Martino A, Esu L et al (2012) Increased response-time variability across different cognitive tasks in children with ADHD. J Atten Disord. doi:10.1177/1087054712439419

Banaschewski T, Brandeis D, Heinrich H, Albrecht B, Brunner E, Rothenberger A (2004) Questioning inhibitory control as the specific deficit of ADHD-evidence from brain electrical activity. J Neural Transm 111:841-864. doi:10.1007/s00702003-0040-8

Baumeister S, Hohmann S, Wolf I et al (2014) Sequential inhibitory control processes assessed through simultaneous EEG-fMRI. Neuroimage 94:349-359. doi:10.1016/j.neuroimage.2014.01.023

Blasi G, Goldberg TE, Weickert T et al (2006) Brain regions underlying response inhibition and interference monitoring and suppression. Eur J Neurosci 23:1658-1664. doi:10.1111/j.14609568.2006.04680.x

Castellanos FX, Sonuga-Barke EJ, Scheres A, Di Martino A, Hyde C, Walters JR (2005) Varieties of attention-deficit/hyperactivity disorder-related intra-individual variability. Biol Psychiatry 57:1416-1423. doi:10.1016/j.biopsych.2004.12.005

Di Martino A, Ghaffari M, Curchack J et al (2008) Decomposing intra-subject variability in children with attention-deficit/hyperactivity disorder. Biol Psychiatry 64:607-614. doi:10.1016/j. biopsych.2008.03.008

Feige B, Biscaldi M, Saville CW et al (2013) On the temporal characteristics of performance variability in attention deficit hyperactivity disorder (ADHD). PLoS ONE 8:e69674. doi:10. 1371/journal.pone.0069674

Fornito A, Zalesky A, Bassett DS et al (2011) Genetic influences on cost-efficient organization of human cortical functional networks. J Neurosci 31:3261-3270. doi:10.1523/jneurosci.485810.2011

Fox MD, Raichle ME (2007) Spontaneous fluctuations in brain activity observed with functional magnetic resonance imaging. Nat Rev Neurosci 8:700-711. doi:10.1038/nrn2201

Kofler MJ, Rapport MD, Sarver DE, Raiker JS, Orban SA, Friedman LM, Kolomeyer EG (2013) Reaction time variability in ADHD: a meta-analytic review of 319 studies. Clin Psychol Rev 33:795-811. doi:10.1016/j.cpr.2013.06.001

Kratz O, Studer P, Malcherek S, Erbe K, Moll GH, Heinrich H (2011) Attentional processes in children with ADHD: an event-related potential study using the attention network test. Int J Psychophysiol 81:82-90. doi:10.1016/j.ijpsycho.2011.05.008

Lazzaro I, Anderson J, Gordon E, Clarke S, Leong J, Meares R (1997) Single trial variability within the P300 $(250-500 \mathrm{~ms})$ processing window in adolescents with attention deficit hyperactivity disorder. Psychiatry Res 73:91-101

Leth-Steensen C, Elbaz ZK, Douglas VI (2000) Mean response times, variability, and skew in the responding of ADHD children: a response time distributional approach. Acta Psychol (Amst) 104:167-190 
MacDonald SW, Nyberg L, Backman L (2006) Intra-individual variability in behavior: links to brain structure, neurotransmission and neuronal activity. Trends Neurosci 29:474-480. doi:10. 1016/j.tins.2006.06.011

Moore RD, Wu CT, Pontifex MB et al (2013) Aerobic fitness and intra-individual variability of neurocognition in preadolescent children. Brain Cogn 82:43-57. doi:10.1016/j.bandc.2013.02. 006

Nakata H, Sakamoto K, Kakigi R (2012) The relationship between reaction time and response variability and somatosensory No-go potentials. Eur J Appl Physiol 112:207-214. doi:10.1007/ s00421-011-1973-5

Penttonen M, Buzsaki G (2003) Natural logarithmic relationship between brain oscillators. Thalamus Relat Syst 2:145-152

Plichta MM, Wolf I, Hohmann S et al (2013) Simultaneous EEG and fMRI reveals a causally connected subcortical-cortical network during reward anticipation. J Neurosci 33:14526-14533. doi:10. 1523/JNEUROSCI.0631-13.2013

Reinhart RM, Mathalon DH, Roach BJ, Ford JM (2011) Relationships between pre-stimulus gamma power and subsequent P300 and reaction time breakdown in schizophrenia. Int J Psychophysiol 79:16-24. doi:10.1016/j.ijpsycho.2010.08.009
Saville CW, Dean RO, Daley D, Intriligator J, Boehm S, Feige B, Klein C (2011) Electrocortical correlates of intra-subject variability in reaction times: average and single-trial analyses. Biol Psychol 87:74-83. doi:10.1016/j.biopsycho.2011.02.005

Segalowitz SJ, Dywan J, Unsal A (1997) Attentional factors in response time variability after traumatic brain injury: an ERP study. J Int Neuropsychol Soc 3:95-107

Sonuga-Barke EJ, Castellanos FX (2007) Spontaneous attentional fluctuations in impaired states and pathological conditions: a neurobiological hypothesis. Neurosci Biobehav Rev 31:977-986. doi:10.1016/j.neubiorev.2007.02.005

Stuss DT, Murphy KJ, Binns MA, Alexander MP (2003) Staying on the job: the frontal lobes control individual performance variability. Brain 126:2363-2380. doi:10.1093/brain/awg237

West R, Murphy KJ, Armilio ML, Craik FI, Stuss DT (2002) Lapses of intention and performance variability reveal age-related increases in fluctuations of executive control. Brain Cogn 49:402-419

Zuo XN, Di Martino A, Kelly C et al (2010) The oscillating brain: complex and reliable. Neuroimage 49:1432-1445. doi:10.1016/j. neuroimage.2009.09.037 